\title{
Nutraceutical supplement in the management of tendinopathies: a systematic review
}

\author{
Federico Fusini ${ }^{1}$ \\ Salvatore Bisicchia ${ }^{2}$ \\ Carlo Bottegoni ${ }^{3}$ \\ Antonio Gigante ${ }^{3}$ \\ Fabio Zanchini ${ }^{1}$ \\ Alberto Busilacchi ${ }^{3}$
}

1 Clinical Orthopaedics, Department of Orthopaedics, Traumatology, Plastic Surgery and Rehabilitation, Second University of Naples, Naples, Italy

2 Department of Orthopaedic Surgery, San Pietro

Fatebenefratelli Hospital, Rome, Italy

3 Department of Clinical and Molecular Sciences, Università Politecnica delle Marche, Ancona, Italy

Corresponding author:

Federico Fusini

Clinical Orthopaedics, Department of Orthopaedics,

Traumatology, Plastic Surgery and Rehabilitation,

Second University of Naples

Via de Crecchio, 2

80138 Naples, Italy

E-mail: fusinif@hotmail.com

\section{Summary}

Background: nutraceuticals are common support therapy for management of tendinopathies. Even if they are widely diffused, our knowledge is still poor. The aim of this systematic review is to analyze the most commonly used nutraceuticals and their effects on tendons.

Methods: glucosamine and chondroitin sulphate, vitamin $\mathrm{C}$, hydrolazed type 1 collagen, arginine alpha-keto-glutarate, bromelain, curcumin, boswellic acid, and methil-sulfonil-methane were considered. During the last week of Dicember 2015 a comprehensive research of main databases for each substance was made in relation with tendinopathy. Repeated articles, articles not in English nor in Italian, not common nutraceuticals, and articles not related with tendons or tenocytes were excluded. Clinical article quality was assessed independently by two reviewers using the modified Coleman methodology score.

Results: preclinical and clinical data from 46 articles from all databases were analyzed. All these nutraceuticals demonstrated several effects on normal and pathological tendons. Preclinical and clinical studies showed a possible role on collagen synthesis, inflammation, mechanical properties, and maturation of collagen bundles, antioxidant effect, edema, and analgesia. The majority clinical studies had some methodological limitations with an average Modified Coleman Methodology Score of 51.3 points and SD of 20.5 points. In particular, there were very low values in power, error, outcome assessment, and clinical effect.

Conclusion: preclinical results are very encouraging, however they are not fully confirmed by clinical studies. There are few clinical papers on the use of nutraceuticals in tendon disorders, and their methodological quality is poor. Furthermore, in most of the studies more than one supplement was administered at the same time. This may bias the results, and the effect of each single component cannot be determined. Furthermore, the interactions between nutraceuticals and drugs, or other dietary supplements (especially at high doses) has not been evaluated, neither their effects on chronic diseases. For these reasons, it is not possible to draw any definitive raccomendations on the use of nutraceutical supplementation in tendinopathies.

KEY WORDS: ascorbate, boswellic acid, curcumin, arginine alpha-keto-glutarate, oral supplement, tendon disorder.

\section{Introduction}

Tendinopathies are common diseases with about $30 \%$ of all consultations with a general practitioner for musculoskeletal disorders. In the last years, the increase of sport activities, life expectancy, and other factors such as environment, diet, systemic diseases and some drug therapies have led to a rise in the incidence of tendinopathies. In fact, not only athletes, but also the general and elder populations suffer from inflammatory or degenerative tendinopathies. In each of these clinical categories, there are 2 common anatomical and functional actors: the quality of tendon tissue and mechanical overuse ${ }^{1}$.

Some tendons suffer more from tendinopathies than others; in particular supraspinatus and biceps brachi tendons, forearm extensor and flexor tendons, patellar tendon, Achilles and tibialis posterior tendons are the most commonly afflicted. 
Various conservative strategies have been proposed to treat tendinopathies and prevent their recurrence ${ }^{2}$. In the last few years the use of oral supplements has been proposed to support the physiological turnover of tendon tissue, in order to prevent inflammation and degeneration. The "teno-protection" is commonly practiced by rheumatologists, orthopaedists, and family physicians. Proponents of these treatments suggest that oral supplements of glucosamine and chondroit in sulphate $(\mathrm{GlcN}-\mathrm{CS})^{3-9}$, vitamin $\mathrm{C}$ (vit C) $)^{10-17}$, hydrolyzed type 1 collagen $(\mathrm{Col} 1)^{18}$, L-arginine alpha-keto-glutarate $(A A K G)^{19-29}$, curcumin ${ }^{30-34}$, boswellic acid $(B A)^{35-38}$, methylsulfonylmethane $(\mathrm{MSM})^{39-41}$, and bromelain ${ }^{42-44}$ increase the concentration of these compounds in tendon context and may help to preserve, or even repair, the damaged tendons. As food supplements, these are not subjected to rigorous controls and licensing processes as drugs; even if some of these products received permissions to be commercialized as drugs in many countries. An amount of pre-clinical studies and randomized controlled trials (RCTs) have been conducted to assess the effectiveness of oral supplements in the management of tendinopathies.

The goal of this systematic review is to report and criticize the current concepts on nutraceutical supplementation in the treatment of tendinopathies.

\section{Materials and methods}

The search was conducted according to the Preferred Reporting Items for Systematic reviews and MetaAnalyses (PRISMA) guideline ${ }^{45}$. With our research, we wished to ascertain the role of nutraceutical elements as support therapy for tendinopathies.

\section{Source of studies and search strategy}

During the last week of Dicember 2015 a comprehensive research of Pubmed, Cochrane and Wiley Library databases was made using various combinations of the names of each substance (glucosamine and chondroitin sulphate, vitamin $\mathrm{C}$ or ascorbate, hydrolyzed type 1 collagen, L-arginine alpha-keto-glutarate, curcumin, boswellic acid, methylsulfonylmethane, and bromelain) and the keywords "nutraceutical", "tendon", "tenocyte", "oral supplement", "tendinopathy".

\section{Study selection and eligibility criteria}

Only original articles were selected, and titles and abstracts were reviewed. The full text was retrieved and reviewed if the abstract suggested that this might be a study in our topic of interest. Article reference lists from general reviews, systematic reviews, and metaanalyses obtained from the search were examined in order to find any other original research investigation not otherwise obtained. In addition, the reference lists of the articles that met the inclusionary criteria were searched. Publications in English and Italian were in- cluded and the research was divided into preclinical and clinical studies. All articles not related to tendons, tendinopathies or tenocytes, all repeated articles and not-common nutraceuticals were excluded.

\section{Quality assessment}

Clinical article quality was assessed independently by two reviewers using the Modified Coleman Methodology Score ${ }^{46}$. In many cases the Authors of each paper did not specifically report all the data necessary to determine the score (i.e. error, power, clinical effect, etc.); in such a case, data were extracted (if possible) and calculated by two independent Authors of the current manuscript. Disagreements between these two assessors were resolved by discussion. Number of patients to treat was not calculated (even if possible) because reporting such a datum is a requirement of the score.

This article meets the ethical standards of the Jour$\mathrm{nal}^{47}$.

\section{Results}

After database search, 865 records were identified. After duplicates removal and study screening, 71 fulltext articles were selected. According to the inclusion and exclusion criteria, 46 articles were included. Results were divided into pre-clinical (40 articles) and clinical studies (6 articles). The corresponding flow diagram is shown in Figure 1.

\section{Preclinical studies}

Glucosamine and chondroitin sulphate Bovine tenocytes and ligament cell colture can be stimulated by the addition of a combination of glucosamine (GlcN) and chondroitin sulphate (CS) to the colture medium. Physiological levels of GICN + CS increased collagen synthesis by $69 \%$ in ligament cells and by $22 \%$ in tenocytes. After $48 \mathrm{~h}$ exposure to 5 $\mu \mathrm{g} / \mathrm{ml} \mathrm{GlcN}+4 \mu \mathrm{g} / \mathrm{ml} \mathrm{CS}$ ligament, epitenon and tenocyte cells reacted with a greater use of hydroxyproline ${ }^{3}$.

Also a horse superficial digital flexor tendon model, treated with bacterial collagenase to simulate an inflammatory process, showed that the injection of glycosaminoglycan polysulphate (GAGPS) promoted the restoration of more organized collagen bundles compared to the control group treated with saline solution ${ }^{4}$.

From a histological point of view, tenotomized rat treated with oral GIcN and CS supplements showed more organized collagen bundles and less inflammation than controls fed with placebo. At eight weeks, rats feeded with GIcN-CS had greater tendon strength in biomechanical tests. A possible explanation for this phenomenon could be the decrease of inflammation and stimulation of collagen synthesis ${ }^{5}$. Similarly, the topical application of CS in the first healing phase of a collagenase-induced injury of rab- 


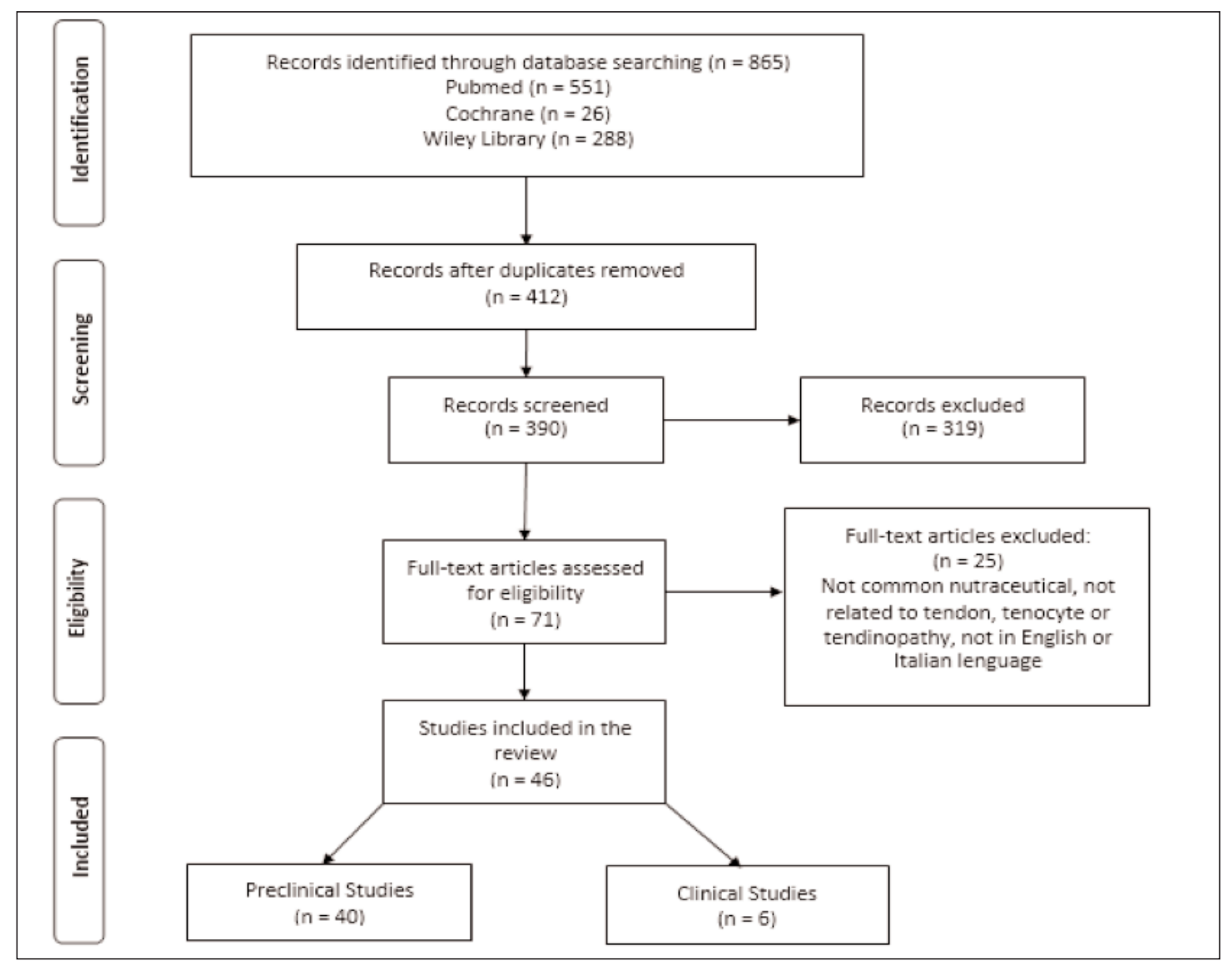

Figure 1. Flow diagram of study selection.

bit's tendons would prevent the degenerative effects of proteases and inflammation on tendon structure and it would preserve tendon composition, morphology, and biomechanical properties. Treated rabbits presented normal orientation of collagen fibrils and tenoblasts compared to untreated animals, which presented a disorganized scar and a higher degree of cellularity. Microscopically, collagen fibrils of treated animals appeared as a mixture of well-preserved large diameter old fibrils and new small diameter regenerated fibrils aligned to the long axis of the tendon. On the other hand, untreated animals presented the predominance of new small randomly arrenged fibrils. Macroscopically, treated tendons returned to normal diameters while untreated injured tendons showed thicker diameters. Treated tendons roughly reached dry weight percentage and collagen contents of control group, whereas those in untreated tendons were significantly lower than control values ${ }^{6,7}$.

CS moreover appeared to protect tendon fascicles from fatigue effects during cycling and static loading regimes, possibly by enabling simple hydration ${ }^{8}$.

In a rabbit experiment of tendon-to-bone healing model, the adjunct of $210-250 \mathrm{mg} / \mathrm{kg} /$ day of GlcN sulphate and $170-200 \mathrm{mg} / \mathrm{kg} /$ day of CS, or placebo showed some interesting results. Extensor digitorum longus tendons were reattached into a bone tunnel in the proximal tibia. Treated tendons showed significantly higher histological scores at 12 weeks. The biomechanical analysis revealed a higher rupture point for treated tendons. However, the difference between the 2 groups was not significant. Moreover, no treated tendon stripped from bone tunnel and all rupture points were within tendon body or at the myotendinous junction ${ }^{9}$.

\section{Vitamin C/ascorbate}

Several studies from 1975 until today suggested that vitamin $\mathrm{C}$ is a crucial factor in the synthesis of procollagen chains, as co-enzime of proline hydroxylase.

The first who studied ascorbate in a cellular model was $\mathrm{Kao}$. He showed that vitamin $\mathrm{C}$ is a valid inductor of procollagenhy droxyproline synthesis in tenocytes of chick embryos. However, the role of ascorbate must be seen as a cofactor of hydroxylation and it does not increase of the activity of prolyl-hydroxylase $^{10}$.

Also primary avian tendon showed that the presence of adequate levels of vitamin $\mathrm{C}$ is fundamental for a normal synthesis of collagen. Infact, tenocytes cultured in a medium without ascorbate synthesized col- 
lagen at only a third of the normal level. Addition of ascorbate to primary avian tendon cells increased, already after about 4 hours, procollagen translation by 6 times, reaching a maximum level after 72 h. The procollagen secretion reacted to ascorbate adjunct with a 6-time change too. This ended up in a reduction of the procollagen half-life within the cell from 120 to $20 \mathrm{~min}$. In sharp contrast to other steps in the procollagen pathway, the change in the secretion rate constant was extremely fast, occurring in less than 30 min. Moreover, the addition of ascorbate to colture medium could speed up the secretion of intracellular procollagen ${ }^{11}$.

However, vitamin $\mathrm{C}$ alone is not able to increase collagen synthesis and secretion. In fact, tenocytes treated with ascorbate, maintained a large pool of procollagen despite changes in translation or secretion rate. This results are compatible with a possible feed-back mechanism between the levels of internal procollagen pool and the rates of collagen synthesis and secretion ${ }^{12}$.

Furthermore, chicken tendon biopsies, daily treated with fresh ascorbate $(0.1 \mathrm{mM})$, appeared to have a significant greater fibroblast migration than controls without ascorbate. Cellular proliferation, collagen synthesis, and total protein synthesis were not significantly altered by ascorbate treatment ${ }^{13}$.

Moreover, ascorbic acid, as an anti-oxydative agent, seemed to enhance the mechanical and thermal stability of rat tail tendon, especially reducing aldimine links, associated with oxidative stress ${ }^{14}$.

The same effect could be seen in a chicken model of flexor tendon injury. Vitamin $\mathrm{C}$ reduced the oxidative stress and the adhesion formation between tendon and surrounding tissues. Infact, vitamin C increased reduced glutathione (GSH) tissue quantity two weeks after injury, in opposition with a control group treated with saline solution. The treatment with ascorbate also significantly improved the gliding resistance of tendons and, after six weeks, it trended to reduce the fibrotic scar at the site of injury. In accordance with macroscopic evaluation, histological exam showed that vitamin $C$ affected the peritendinous adhesion of treated tendons, making them less likely to be formed, than in control group ${ }^{15}$.

The experiment of Russel et al. in 1991 aimed to establish the optimal levels of ascorbic acid to boost tendon healing. They prepared a tenocyte colture from profundus flexor tendons of New Zealand white rabbits with 50-300 micrograms $/ \mathrm{ml}$ of ascorbic acid, added fresh every 48 hours medium change. Levels of ascorbate in colture medium were strictly related with tendon collagen and non collagen protein synthesis, glucose metabolism, and cell permeability/viability. Their results suggested that more than the traditional 50 micrograms $/ \mathrm{ml}$ of ascorbic acid are necessary to optimally maintain tenocytes in culture with 48 hour medium and ascorbate changes ${ }^{16}$.

A recent study in 42 healthy female Wistar Albino rats proved that high-dose vitamin $C$ supplementation every 2 days could have stimulating effects on healing of a full-thickness Achilles tendon ruptures be- cause of early angiogenesis and collagen synthesis improvement ${ }^{17}$.

\section{Hydrolyzed type 1 collagen}

The role of collagen 1 on tenocytes or tenoblasts colture is still little investigated.

A study based on the investigation of the effects of collagen peptides ingestion on the extracellular matrix of tendon, demonstrates that hydrolyzed type 1 collagen peptides affect the size of collagen fibrils and composition of glycosaminoglycans in Achilles tendon. The ingestion of type 1 collagen increased the amount of dermatansulphate and lowered the percentage of hyaluronic acid, especially if hydrolyzed collagen was administered at high dose. The rise of this biochemical component may ameliorate the mechanical properties of Achilles tendon, at least in a rabbit model ${ }^{18}$.

\section{L-arginine-alfa-keto-glutarate}

L-arginine is an essential amino acid required by the enzymatic family of nitric-oxide-synthase (NOS) to produce nitric oxide (NO) ${ }^{19}$ which is a crucial factor in tendon healing process ${ }^{20}$. Infact, in a rat model uninjured tendons did not present NOS activity and immune reactivity. After surgical tenotomy, NOS enzyme increased its activity and immune reactivity by 5 times, reaching a maximum after 7 days, with a reduction near baseline levels at day $14^{21}$. Inhibition of NOS activity with oral administration of an analogue of L-arginine - $\mathrm{N} \omega$-nitro-L-arginine methyl ester ( $\mathrm{L}$ NAME) - was possible, leading to a significant reduction of cross sectional area and failure load of injured Achilles tendons ${ }^{22}$.

Different NOS isoforms are activated at different time points. In another study, some days after injury, there was an increase of mRNA levels for each NOS isoform, with the peak of inducible isoform (iNOS) at day 4 , endothelial isoform (eNOS) at day 7 and neuronal isoform (bNOS) at day 21. All three NOS isoforms altered their levels during the healing process with differential patterns of temporal expression during the various phases of tendon healing ${ }^{23}$.

In animal models, the addition of competitive NOS inhibitors reduced tendon healing, while the addition of NO enhanced tendon healing 24 .

The levels of Trasforming Growth Factor (TGF) $\beta$ undergo a change during physiological repair process. At the beginning they rise and after about 3 weeks they gradually decrease to slowly reach control levels. However, if L-NAME is given at injury time, the macrophage infiltrates continue to express high level of TGF- $\beta$ and this persists also after 5 weeks from injury, with no tendency to reduction. Following an injury, iNOS activity is the highest during the acute phase of inflammation, and then gradually trends to reduce to normal. Treatment of injured tendon with LNAME, however, is able to inhibit iNOS activity at 3 days and to reduce enzymatic activity at later time. LNAME-treated rats showed increased levels of adhe- 
sion of peritoneal macrophages to epitenon monolayers in vitro, which is the demonstration of the chronic disequilibrium between the over expression of TGF- $\beta$ and the under expression of NO. In addition the treatment of acute tendon injury with NO inhibitor causes a long-lasting and wide accumulation of many inflammatory cells in the subcutaneous tissue, muscle and tendon as a response of chronic inflammation ${ }^{25}$. Thus, the formation of NO is a key event for tendon healing process because its inhibition increases the level of TGF- $\beta^{26}$ and development of fibrosis and chronic inflammation ${ }^{27}$.

The deposition of collagen is a key event during wound healing. The addiction of L-arginine to cultured human tenocytes, increasing nitric oxide production, can enhance and promote collagen synthesis ${ }^{28}$. However, Moraes et al. demonstrated that local treatment with L-NAME significantly improved the functional parameters and accelerated histomorphological recovery of injured murine Achilles tendons ${ }^{29}$.

\section{Curcumin}

Curcumin is an antioxidant extracted from Curcuma Longa that showed a good potential in the prevention of oxidative stress damage.

Oxidative stress, accumulation of lipid peroxidation products, and accelerated accumulation of advanced glication end product of collagen (AGE-collagen), expecially in diabetic serum are reduced significantly by curcumin administration. Extensive cross-linking of collagen bundles with disruption of molecular structure was also prevented to a greater extent by curcumin treatment ${ }^{30}$.

Increased vascularization is a very common finding encountered in case of tendinopathy, Curcumin, inhibiting the formation of new blood vessels, can be useful in the treatment of this pathology. Inhibition of endothelial cells proliferation, whether basic fibroblast growth factor (bFGF) is present or not, seems to be another ability of curcumin as well as its ability to inhibit proliferation of immortalized endothelial cell. In a mouse model, curcumin effectively inhibited endothelial cell proliferation in a dose-dependent manner demonstrating a significant inhibition of bFGF-mediated neovascularization ${ }^{31}$.

Long period of oral administration of curcumin decreased the accumulation and cross-linking of collagen, bringing it back to its original characteristic ${ }^{32}$. Wide advanced glycation products, precipitation of collagen salt, and alteration of its acid and pepsin digestion are common findings in tendinopathies and the administration of curcumin is able to restore tendon characteristics ${ }^{32}$.

Curcumin targets NF-KB signaling pathway. The concentrations of $5-20 \mu \mathrm{M}$ of curcumin inhibit inflammation and apoptosis induced by IL-1 $\beta$ in vitro. Curcumin performs its anti-inflammatory effects with the down-regulation of matrix metalloproteinase-1, -9 and -13 , cyclooxygenase-2, the inhibition of caspase- 3 and Bax pathway leading to apoptosis, and with the stimulation of cell survival promoting Bcl-2 pathway.
In addition, curcumin inhibits kB-kinase activity, suppressing the degradation of $\mathrm{kBa}$ inhibitor, and inhibition of nuclear traslocation of NF-KB, all related to IL$1 \beta$-induced NF-KB activation. Moreover, curcumin seems to play some role in the phosphatidylinositol 3kinase (PI-3K) p85/Akt pathway ${ }^{33}$.

Jiang et al. induced patellar tendon injury in SpragueDawling rats and tested oral administration of curcumin (100 mg/kg of body weight). To evaluate the healing process, Authors analysed gene expression of tenocyte-related genes, malonildialdehyde (MDA) levels, manganese-dependent superoxide dismutase (MnSOD) activity, hydroxyproline and biomechanical tests. Histological examination of treated animals showed deposition of well-organized collagen fibers, lower level of MDA and increase of biomechanical properties along with increase MnSOD activity than the same outcomes of control group ${ }^{34}$.

\section{Boswellic acid}

Since 2002 Boswellic acid (BA), extracted from Boswellia Serrata, proved to be an active treatment for chronic inflammatory diseases. Although BA is often used in association with other nutraceuticals in the treatment of tendinopathies, no specific articles citing the effects of BA on tenocytes were found with the current search strategy.

Certain BA shave been described to inhibit proliferation and to induce the apoptosis of leukocytes and to depress elastase enzyme activity.

BAs are able to inhibit 5-lipoxygenase and leukotriene biosynthesis in neutrophilic granulocytes ${ }^{35}$. Animal experiments showed the anti-inflammatory activity of Boswellia extract, and this mechanism is related to immune system component, unlike NSAIDs; the main action is the inhibition of 5-lipoxygenase, but also other factors such as cytokines (interleukines and TNFa), complement system, leukocyte elastase and oxygen radicals are involved ${ }^{36}$. BA also prevents the TNF a-induced expression of matrix metalloproteinases MMP-3, MMP-10, and MMP-12 ${ }^{37}$.

$\mathrm{BA}$ avoids NF-KB pathway activation by neutrophilic granulocytes, and consequently it down regulates proinflammatory cytokines such as TNF-a, IL-1, IL-2, IL-4, IL-6 and IFN- $\gamma$. BA is also able to influence the classic way of the complement system suppressing the conversion of $\mathrm{C} 3$ into $\mathrm{C} 3 \mathrm{a}$ and $\mathrm{C} 3 \mathrm{~b}$. Only little role on global inflammation is played by the BA interaction with prostaglandin synthesis. Another interesting characteristic of BA is the inhibitory action against 5-LO that leads to a decrease of the production of leukotrienes, since several chronic inflammatory deseases are associated with an enhancement of leukotrienes activity.

However, 11-keto- $\beta$ BA and acetyl-11-keto- $\beta$ BA seem not able to inhibit the production of leukotrienes in human blood, and pharmacokinetic data reveal very low plasmatic concentrations of them, not adequate to reach the same effective concentrations for bioactivity in vitro ${ }^{36}$.

Nonetheless, $\beta$-BA has 100-fold higher plasma con- 
centrations than other BAs, and this concentration is enough to inhibit microsomal prostaglandin E synthase- 1 and the serine protease cathepsin G. These two enzymes might be reasonable molecular targets related to the anti-inflammatory properties of Boswellia Serrata extract ${ }^{38}$.

\section{Methylsulfonilmethane (MSM)}

Methylsulfonylmethane (MSM) is a common oral supplement used in wide range of morbid conditions such as pain, inflammation, allergies, arthritis, parasitic and bacterial infections, etc. ${ }^{39}$.

This sulfur-containing compound can be found in a variety of human food like vegetables, grains, fruits, and beverages, and the oral consumption can be rapidly absorbed, well distributed, and completely excreted from the body 40 .

It is a natural substance with efficacious analgesic and anti-inflammatory properties and its use in the management of osteoarthritis demonstrated to be effective and rapid to reduce pain, swelling and to improve the functional ability of joints ${ }^{41}$.

No studies about MSM on tenocytes were found with the current search strategy.

\section{Bromelain}

Several studies since 1964 demonstrated that bromelain has a pharmacological potential in the treatment of edema and ecchymosis following surgical and nonsurgical trauma. Bromelain is an ensemble of proteases derived from pineapple stem with some therapeutic effects in several inflammatory diseases.

In vitro, experiments have shown that bromelain was able to remove several essential cell surface molecules for leukocyte trafficking, such as CD128a/CXCR1 and CD128b/CXCR2, and served as receptors for IL-8. Anyway, these effects did not affect whole leukocyte migration. Infact, the response to bacterial antigen remained preserved. In vivo, bromelain treatment reduced from 50 to $85 \%$ neutrophilic granulocytes migration, temporarily depressing leukocyte rolling, inhibiting the strong adhesion of leukocytes to vascular endothelium around the site of inflammation. In response to adhesion changes, leucocytes had a fast re-expression of the bromelain-sensitive CD62L/L-selectin molecules which allow rolling after bromelain administration and a minimal re-expression of CD128 after some time ${ }^{42}$.

Not all the parts of pineapple have the same properties against tenocytes and oxidative stress. Aiyegbusi et al. observed that the flesh and bark extract of pineapple plant, which contains the enzyme bromelain, promoted murinetenoblast proliferation. Contrariwise, leaves and core extracts reduced tenocytes population. Leaves and core extracts significantly increased MDA, which is produced during the early phase of Achilles tendon healing process after injury, and can be considered a marker of tendon oxidative stress levels, while flesh extract reduced those levels. The bark extract seemed to have no significant im- pact on MDA level compared with untreated tendons, suggesting that flesh contains the anti-oxidant substances of pineapple plant while the potential role to promote healing by stimulating tenoblast proliferation could be due to bark and flesh extracts ${ }^{43}$.

Comparing the effects of fresh pineapple juice with commercial bromelain on tenocyte proliferation and the MDA levels in the first stage of Achilles tendon healing after injury, Aiyegbusi observed that tenocyte population of bromelain group was significantly larger than control group, while also tenocytes of fresh pineapple juice group rised their number, although not significantly. Pineapple juice, however, significantly lowered the MDA level compared with both the control and bromelain-treated group ${ }^{44}$.

\section{Nutraceutical formulation}

A recent study on human primary tenocytes isolated from finger tendon of male middle-age donors has suggested that a supplementation with a commercial nutraceutical supplement, named TENDOACTIVE ${ }^{\text {TM }}$ (Bioiberica S.A., Palafolls, Spain), containing mucopolysaccharides, hydrolyzed type 1 collagen, and vitamin $C$, could suppress the activation of NF-kB induced by $\mathrm{IL}-1 \beta$, and the nuclear translocation of $p 65$. These events are related with the down-regulation of several targets of NF-kB activation pathway including COX-2, MMP-1, and activated caspase-3. This formulation also prevents the down-regulation of collagen type 1 induced by IL-1 $\beta$ and $\beta 1$-integrine receptor expression $^{48}$.

\section{Clinical studies}

The management of tendinopathies with oral supplements is a relative new approach; for this reason the lack of clinical studies about nutraceutical supplements does not allow a specific evaluation for each substance. The current clinical studies often test more than one substance at the same time and they often analyze pharmaceutical preparation or the combination of oral supplement with physical activities or other treatments.

A clinical study, comparing the effects of Glc-Nor indomethacin administration in the management of Achilles peritendinitis showed that Glc- $\mathrm{N}$ had a better overall therapeutic effect on $2 / 3$ of the patients compared to $1 / 3$ of the patients treated with Indomethacin. Especially in those patients who endure a persistent pain, Glc-N proved to be more effective than indomethacin. Also non-responders to Indomethacin showed a little/moderate benefit from Glc-Ntherapy on pain level ${ }^{49}$.

Oral supplementation of hydrolyzed type 1 collagen, arginine L-alpha-chetoglutarate, MSM, and bromelain has a potential benefic role in tendon healing, lowering the pain due to tendinopathy. It demonstrated to be a valuable tool in human arthroscopic rotator cuff tear repair. Gumina et al. reported that nutraceutical supplementation for 3 months after rotator cuff repair lowered post-operative shoulder pain and led to a 
slight improvement in repair integrity. Despite these results, it is not possibile to find a better objective functional outcome related with this improvement. According to the Authors, these effects could help and shorten the physical rehabilitation program and prevent new re-ruptures ${ }^{50}$; however there are some limitations, such as a low number of patients and a relatively short follow up.

Notarnicola et al. tested if extracorporeal shockwave therapy produced good results in the treatment of insertional Achilles tendinopathy. Its efficacy can be boosted with administration of dietary supplements containing L-arginine-a-cheto-glutarate, vinitrox ${ }^{\mathrm{TM}}$ (a polyphenolic compound), MSM, bromelain, type 1 collagen, and vitamin C. The effect of nutraceutical supplementation and extracorporeal shockwave therapy was studied in patients with insertional Achilles tendinopathy and the Authors reported that the combined treatment lead to a lower level of pain and better results at Ankle-Hindfoot scale, and Roles and Maudsley score than extracorporeal shockwave therapy alone ${ }^{51}$.

Even if Nakhostin-Roohi studied the reductive potencial of MSM after an acute exhaustive exercise, this clinical trial seemed to support the hypothesis that also a single dose of MSM could increase the level of total antioxidant capacity. MSM administration also lower the values of serum MDA, uric acid, protein carbonyl (PC) as a marker of oxidative stress, which could happen in case of tendon disorders ${ }^{52}$.

In the last year one study evaluated the commercial available dietary supplement TENDOACTIVETM (Bioiberica S.A., Palafolls, Spain) composed by mucopolisaccharides, type 1 collagen, and vitamin $C$ in the management of different tendinopathies (Achilles, patellar, and common extensor tendons). The overall results seemed to show an improvement of symptoms and structural evolution of injured tendons. In fact, patients treated with oral supplementation for 90 days showed a reduction of pain and a functional improvement already after 10 days. Each type of tendinopathy showed at day 90 a significant improvement of functional scales: $38 \%$ for Achilles, $46 \%$ for patellar and $77 \%$ for common extensor tendons, and also a reduction of tendon thickness from 10 to $20 \%$, depending on the anatomical region ${ }^{53}$.

Merolla et al. assessed the analgesic effect of a dietary compound named TENDISULFUR ${ }^{\circledR}$ (Laborest SpA, Nerviano, Italy) in patients with a full-thickness supraspinatus tendon rupture treated arthroscopically. This oral supplement contains GlcN-CS, vitamin C, type 1 collagen, L-arginine-a-keto-glutarate, BA, curcumin, and MSM. Patients were randomly assigned to dietary supplement or placebo for 2 months. After 1 week, treatment group showed significantly lower level of VAS, night pain and pain after activity. Constant-Murley score and simple shoulder test (SST) did not differ between the 2 groups. Patients also reported a good global assessment and no adverse effects. Already after 2 weeks all scores presented no significant differences, even if pain values were lower. The Authors concluded that TENDISULFUR ${ }^{\circledR}$ alle- viated short and partially mid-term pain, but did not affect long term pain. To solve this limitation, the Authors suggested to increase dosage over the first 4 weeks and by extending treatment by 1 or 2 months ${ }^{54}$.

\section{Methodological quality evaluation for clinical studies}

The majority of studies included in the review had some methodological limitations with an average Modified Coleman Methodology Score of 51.3 points and SD of 20.5 points (Tab. 1). Only 4 out of 6 studies reaches a fair methodological quality score. In particular, there were very low values in power, error, and outcome assessment. The number of patients to treat was never reported in the included studies. Furthermore, sample size calculation has rarely been performed, and in some cases it is not clear who evaluated the patients at enrollement and at follow-up (the treating physician or an independent investigator).

\section{Discussion}

Tendinopathy is a very common disease, and it is still difficult to manage. The risk of recurrence is very high and a resolutive therapy is still not available. Nutraceuticals are widely adopted in clinical practice, in the general and athletic populations, aiming at reducing symptoms from tendinopaties, prevent tendon injuries, and recover earlier from trauma or surgery. As in many other cases, trends in medical practice and drug prescription usually anticipate the availability of the best scientific evidence on that specific treatment. Nutraceuticals are the case, in particular because these "natural" substances have deemed to have no side effects. Even if many prescribed medications and drugs are derived from natural sources (i.e. plants), but this does not make them safe as a definition.

From the search strategy, an amount of preclinical studies about nutraceutical supplementation was retrived demonstrating encouraging results in both human tenocytes and animal models. Even if preclinical studies are usually more rigorous and usually consider each substance independently, the current search strategy was not able to find any preclinical study dealing on the effect of MSM or BA alone on tendons. These substances are videly adopted in clinical practice (in associations with other nutraceuticals in most of the cases), and they are adopted in many of the clinical studies included in the current review41,50,51,54. Despite the evidence from preclinical studies is quite consistent and strong, only 6 clinical studies were obtained from our search strategy. Results from clinical studies tend to confirm preclinical evaluations, but are biased by inclusion of multiple substances in the same formulation and poor methodology. In this systematic review, data extraction and calculation of several items of the Modified Coleman Methodology Score was performed to determine the real quality of the studies. In fact, in many cases the Authors did not specifically report some data (such as percentages), 
but all the information required for calculation was clearly reported. On the other hand, severe methodological errors have been encountered during the reviewing process in many articles; i.e. in sample size calculation, description of primary and secondary end-points, power analysis, follow-up length, blinding, determination of the clinical effect of the treatment (effect size, absolute risk reduction, relative risk reduction), number of patients to treat. Determination of some of these items was not easy because data were confusing and sparsely reported (sometimes only as a graph without reporting the exact value for mean and/or standard deviation), and in some cases it was not possible at all. If the items not clearly reported in each paper were not calculated (if possible) for this systematic review, the Modified Coleman Methodology Score would have been even lower, confirming the poor methodologically quality of the available literature on this topic.

Nutraceuticals are not only used in the general population, but also in athletes. In particular many of them (especially non-professional) may abuse of these substances, having in their mind the idea to improve their results, decrease their recovery time, prevent injuries, or return to sports earlier after trauma or surgery. On the other hand, the safety profile of high doses of nutraceuticals has been rarely questioned, if ever. Furthermore, the interactions between nutraceuticals and drugs, or other dietary supplements (especially at high doses) has not been determined, neither their effects on chronic diseases.
Some limitations of this review have to be acknowledged. In particular, despite a broad searching strategy, very few papers have been included (especially clinical studies), and most of them have low methodological quality. The current search strategy was not able to find any preclinical study on the effect of MSM or BA alone on tendons; probably different keywords would have lead to different results, but this limitation is intrinsic to all systematic reviews.

\section{Conclusions}

Preclinical results are very encouraging, however they are not fully confirmed by clinical studies. There are few clinical papers on the use of nutraceuticals in tendon disorders, and their methodological quality is poor. Furthermore, in most of the studies more than one supplement was administered at the same time. This may bias the results, and the effect of each single component cannot be determined. Furthermore, the interactions between nutraceuticals and drugs, or other dietary supplements (especially at high doses) has not been evaluated, neither their effects on chronic diseases. For these reasons, it is not possible to draw any definitive recommendations on the use of nutraceutical supplementation in tendinopathies, and further studies are required to improve our knowledge on this topic.

A summary of each compound and its properties can be found in Table 1.

Table 1. Overview of principal nutraceutical and their properties (GIC-N-CS: glucosamine and chondroitin sulphate; vit C: vitamin C; Col I: collagen type 1; Col III collagen type 3; AAKG: L-arginine-a-keto-glutarate; NOS: nitric oxide synthase; NO: nitric oxide; 5-LO: 5-lipoxygenase; TNF $\alpha$ : tumor necrosis factor $\alpha$; IL-1/2/4/6: interleukin 1/2/4/6; IFNY: interferon y; MSM: methilsulfonil methane; MDA: malonyldialdehyde; GSSG: oxidized gluthation).

Nutraceutical Biological effect

Glucosamine and chondroitin sulphate (GlcN-CS) Increase collagen synthesis, ameliorate mechanical properties, organization of collagen bundles and resistence to fatigue, helpful in the management of pain.

Vitamin C (Vit C)

Collagen I (Col I)

L-arginine-a-keto-glutarate

Curcumin

Boswellic acid

Methilsulfonilmethane (MSM)
Stimulate hydroxyproline synthesis of procollagen, enhance angiogenesis and maturation of Col III to Col I fibers, anti-inflammatory and antioxidant effect.

Increase mechanical properties, beneficial effects on collagen-rich tissues.

Substrate of NOS, increase NO levels and collagen synthesis.

Neoangiogenesis and apotosis inhibitor, antioxidant effect, stimulate tenocytes survival.

Elastase and 5-LO activity inhibition, reduce TNFa, IL-1, IL-2, IL-4, IL- 6 e INFy levels.

Analgesic, anti-inflammatory and antioxidant effects, reduce MDA and GSSG levels.

Decrease lymphocytes rolling, anti edema, antioxidant and immunosuppressive effects, reduce MDA levels. 


\section{Conflict of interests}

The Authors declare that they have no conflict of interests regarding the publication of this paper.

\section{References}

1. Maffulli N, Wong J, Almekinders LC. Types and epidemiology of tendinopathy. Clinics in sports medicine. 2003;22(4):675692.

2. Maffulli N, Longo UG, Denaro V. Novel approaches for the management of tendinopathy. The Journal of bone and joint surgery American volume. 2010;92(15):2604-2613.

3. Lippiello L. Collagen Synthesis in tenocytes, ligament cells and chondrocytes exposed to a combination of Glucosamine $\mathrm{HCl}$ and chondroitin sulfate. Evidence-based complementary and alternative medicine: eCAM. 2007;4(2):219-224.

4. Moraes JR, Facco GG, Moraes FR, Engracia Filho JR, Miyazato LG, Beretta DC. Effects of glycosaminoglycan polysulphate on the organisation of collagen fibres in experimentally induced tendonitis in horses. The Veterinary record. 2009;165(7):203-205.

5. Ozer H, Taskesen A, Kul O, Selek HY, Turanli S, Kose K. Effect of glucosamine chondroitine sulphate on repaired tenotomized rat Achilles tendons. Eklem hastaliklari ve cerrahisi $=$ Joint diseases \& related surgery. 2011;22(2):100-106.

6. Oryan A, Goodship AE, Silver IA. Response of a collagenaseinduced tendon injury to treatment with a polysulphated glycosaminoglycan (Adequan). Connective tissue research. 2008;49(5):351-360

7. Oryan A, Moshiri A, Meimandiparizi AH. Effects of sodiumhyaluronate and glucosamine-chondroitin sulfate on remodeling stage of tenotomized superficial digital flexor tendon in rabbits: a clinical, histopathological, ultrastructural, and biomechanical study. Connective tissue research. 2011;52(4):329339.

8. Legerlotz K, Riley GP, Screen HR. GAG depletion increases the stress-relaxation response of tendon fascicles, but does not influence recovery. Acta biomaterialia. 2013;9(6):68606866.

9. Taskesen A, Ataoglu B, Ozer M, Demirkale I, Turanli S. Glucosamine-chondroitin sulphate accelerates tendon-to-bone healing in rabbits. Eklem hastaliklari ve cerrahisi $=$ Joint diseases \& related surgery. 2015;26(2):77-83.

10. Kao WW, Flaks JG, Prockop DJ. Primary and secondary effects of ascorbate on procollagen synthesis and protein synthesis by primary cultures of tendon fibroblasts. Archives of biochemistry and biophysics. 1976;173(2):638-648.

11. Schwarz RI, Kleinman P, Owens N. Ascorbate can act as an inducer of the collagen pathway because most steps are tightly coupled. Annals of the New York Academy of Sciences. 1987;498:172-185.

12. Schwarz RI, Mandell RB, Bissell MJ. Ascorbate induction of collagen synthesis as a means for elucidating a mechanism of quantitative control of tissue-specific function. Molecular and cellular biology. 1981;1(9):843-853.

13. Nelson JM, Diegelmann RF, Cohen IK. Effect of beta-aminopropionitrile and ascorbate on fibroblast migration. Proceedings of the Society for Experimental Biology and Medicine Society for Experimental Biology and Medicine. 1988;188(3):346352.

14. Rigby BJ, Mitchell TW. In vitro studies of the interaction between ascorbic acid and rat tail tendon. Biochimica et biophysica acta. 1978;544(1):62-68.

15. Hung LK, Fu SC, Lee YW, Mok TY, Chan KM. Local vitamin-C injection reduced tendon adhesion in a chicken model of flex- or digitorum profundus tendon injury. The Journal of bone and joint surgery American volume. 2013;95(7):e41.

16. Russell JE, Manske PR. Ascorbic acid requirement for optimal flexor tendon repair in vitro. Journal of orthopaedic research: official publication of the Orthopaedic Research Society. 1991;9(5):714-719.

17. Omeroglu S, Peker T, Turkozkan N, Omeroglu H. High-dose vitamin $\mathrm{C}$ supplementation accelerates the Achilles tendon healing in healthy rats. Archives of orthopaedic and trauma surgery. 2009;129(2):281-286.

18. Minaguchi J, Koyama $\mathrm{Y}$, Meguri $\mathrm{N}$, et al. Effects of ingestion of collagen peptide on collagen fibrils and glycosaminoglycans in Achilles tendon. Journal of nutritional science and vitaminology. 2005;51(3):169-174.

19. Kharitonov SA, Lubec G, Lubec B, Hjelm M, Barnes PJ. L-arginine increases exhaled nitric oxide in normal human subjects. Clinical science (London, England : 1979). 1995;88(2):135139.

20. Bokhari AR, Murrell GA. The role of nitric oxide in tendon healing. Journal of shoulder and elbow surgery / American Shoulder and Elbow Surgeons. 2012;21(2):238-244.

21. Murrell GA. Using nitric oxide to treat tendinopathy. British journal of sports medicine. 2007;41(4):227-231.

22. Murrell GA, Szabo C, Hannafin JA, et al. Modulation of tendon healing by nitric oxide. Inflammation research : official journal of the European Histamine Research Society. 1997; 46(1):1927.

23. Lin JH, Wang MX, Wei A, Zhu W, Diwan AD, Murrell GA. Temporal expression of nitric oxide synthase isoforms in healing Achilles tendon. Journal of orthopaedic research: official publication of the Orthopaedic Research Society. 2001;19(1):136142.

24. Murrell GA. Oxygen free radicals and tendon healing. Journal of shoulder and elbow surgery/American Shoulder and Elbow Surgeons. 2007;16(5 Suppl):S208-214.

25. Darmani H, Crossan JC, Curtis A. Single dose of inducible nitric oxide synthase inhibitor induces prolonged inflammatory cell accumulation and fibrosis around injured tendon and synovium. Mediators of inflammation. 2004;13(3):157-164.

26. Darmani H, Crossan J, McLellan SD, Meek D, Adam C. Expression of nitric oxide synthase and transforming growth factor-beta in crush-injured tendon and synovium. Mediators of inflammation. 2004;13(5-6):299-305.

27. Tomiosso TC, Nakagaki WR, Gomes L, Hyslop S, Pimentel ER. Organization of collagen bundles during tendon healing in rats treated with L-NAME. Cell and tissue research. 2009;337 (2):235-242.

28. Xia W, Szomor Z, Wang Y, Murrell GA. Nitric oxide enhances collagen synthesis in cultured human tendon cells. Journal of orthopaedic research: official publication of the Orthopaedic Research Society. 2006;24(2):159-172.

29. Moraes SA, Oliveira KR, Crespo-Lopez ME, Picanco-Diniz DL, Herculano AM. Local NO synthase inhibition produces histological and functional recovery in Achilles tendon of rats after tenotomy: tendon repair and local NOS inhibition. Cell and tissue research. 2013;353(3):457-463.

30. Sajithlal GB, Chithra P, Chandrakasan G. Effect of curcumin on the advanced glycation and cross-linking of collagen in diabetic rats. Biochemical pharmacology. 1998;56(12):16071614.

31. Arbiser JL, Klauber N, Rohan R, van Leeuwen R, Huang MT, Fisher $\mathrm{C}$, et al. Curcumin is an in vivo inhibitor of angiogenesis. Molecular medicine (Cambridge, Mass). 1998;4(6):376-383.

32. Pari L, Murugan P. Influence of tetrahydrocurcumin on tail tendon collagen contents and its properties in rats with streptozotocin-nicotinamide-induced type 2 diabetes. Fundamental \& clinical pharmacology. 2007;21(6):665-671.

33. Buhrmann C, Mobasheri A, Busch F, et al. Curcumin modu- 
lates nuclear factor kappaB (NF-kappaB)-mediated inflammation in human tenocytes in vitro: role of the phosphatidylinositol 3-kinase/Akt pathway. The Journal of biological chemistry. 2011;286(32):28556-28566.

34. Jiang $\mathrm{D}$, Gao $\mathrm{P}$, Lin $\mathrm{H}$, Geng $\mathrm{H}$. Curcumin improves tendon healing in rats: a histological, biochemical, and functional evaluation. Connective tissue research. 2015:1-8.

35. Ammon HP. Boswellic acids (components of frankincense) as the active principle in treatment of chronic inflammatory diseases. Wiener medizinische Wochenschrift (1946). 2002; 152(15-16):373-378.

36. Ammon HP. Modulation of the immune system by Boswellia serrata extracts and boswellic acids. Phytomedicine: international journal of phytotherapy and phytopharmacology. 2010;17(11):862-867.

37. Roy S, Khanna S, Krishnaraju AV, et al. Regulation of vascular responses to inflammation: inducible matrix metalloproteinase-3 expression in human microvascular endothelial cells is sensitive to antiinflammatory Boswellia. Antioxidants \& redox signaling. 2006;8(3-4):653-660.

38. Abdel-Tawab M, Werz O, Schubert-Zsilavecz M. Boswellia serrata: an overall assessment of in vitro, preclinical, pharmacokinetic and clinical data. Clinical pharmacokinetics. 2011;50 (6):349-369

39. Kim YH, Kim DH, Lim H, Baek DY, Shin HK, Kim JK. The antiinflammatory effects of methylsulfonylmethane on lipopolysaccharide-induced inflammatory responses in murine macrophages. Biological \& pharmaceutical bulletin. 2009;32 (4):651-656.

40. Magnuson BA, Appleton J, Ames GB. Pharmacokinetics and distribution of [35S]methylsulfonylmethane following oral administration to rats. Journal of agricultural and food chemistry. 2007;55(3):1033-1038.

41. Usha PR, Naidu MU. Randomised, Double-Blind, Parallel, Placebo-Controlled Study of Oral Glucosamine, Methylsulfonylmethane and their Combination in Osteoarthritis. Clinical drug investigation. 2004;24(6):353-363.

42. Fitzhugh DJ, Shan S, Dewhirst MW, Hale LP. Bromelain treatment decreases neutrophil migration to sites of inflammation. Clinical immunology (Orlando, Fla). 2008;128(1):66-74.

43. Aiyegbusi Al, Duru FI, Awelimobor D, Noronha CC, Okanlawon $\mathrm{AO}$. The role of aqueous extract of pineapple fruit parts on the healing of acute crush tendon injury. Nigerian quarterly journal of hospital medicine. 2010;20(4):223-227.

44. Aiyegbusi AI, Olabiyi OO, Duru FI, Noronha CC, Okanlawon AO. A comparative study of the effects of bromelain and fresh pineapple juice on the early phase of healing in acute crush achilles tendon injury. Journal of medicinal food. 2011;14(4):
348-352.

45. Liberati A, Altman DG, Tetzlaff J, et al. The PRISMA statement for reporting systematic reviews and meta-analyses of studies that evaluate healthcare interventions: explanation and elaboration. BMJ (Clinical research ed). 2009;339:b2700.

46. Coleman BD, Khan KM, Maffulli N, Cook JL, Wark JD. Studies of surgical outcome after patellar tendinopathy: clinical significance of methodological deficiencies and guidelines for future studies. Victorian Institute of Sport Tendon Study Group. Scandinavian journal of medicine \& science in sports. 2000 10(1):2-11.

47. Padulo J, Oliva F, Frizziero A, Maffulli N. Muscles, Ligaments and Tendons Journal. Basic principles and recommendations in clinical and field science research. MLTJ. 2013;3(4):250252.

48. Shakibaei M, Buhrmann C, Mobasheri A. Anti-inflammatory and anti-catabolic effects of TENDOACTIVE(R) on human tenocytes in vitro. Histology and histopathology. 2011;26(9): 1173-1185.

49. Sundqvist $H$, Forsskahl $B$, Kvist M. A promising novel therapy for Achilles peritendinitis: double-blind comparison of glycosaminoglycan polysulfate and high-dose indomethacin. International journal of sports medicine. 1987;8(4):298-303.

50. Gumina S, Passaretti D, Gurzi MD, Candela V. Arginine L-alpha-ketoglutarate, methylsulfonylmethane, hydrolyzed type I collagen and bromelain in rotator cuff tear repair: a prospective randomized study. Current medical research and opinion. 2012;28(11):1767-1774.

51. Notarnicola A, Pesce V, Vicenti G, Tafuri S, Forcignano M, Moretti B. SWAAT study: extracorporeal shock wave therapy and arginine supplementation and other nutraceuticals for insertional Achilles tendinopathy. Advances in therapy. 2012;29 (9):799-814.

52. Nakhostin-Roohi B, Niknam Z, Vaezi N, Mohammadi S, Bohlooli S. Effect of single dose administration of methylsulfonylmethane on oxidative stress following acute exhaustive exercise. Iranian journal of pharmaceutical research: IJPR 2013;12(4):845-853.

53. Arquer A, García M, Laucirica JA, et al. Eficacia y seguridad de un tratamiento oral a base de mucopolisacáridos, colágeno tipo i y vitamina $C$ en pacientes con tendinopatías. Apunts Medicina de l'Esport. 2014;49(182):31-36.

54. Merolla G, Dellabiancia F, Ingardia A, Paladini P, Porcellini G. Co-analgesic therapy for arthroscopic supraspinatus tendon repair pain using a dietary supplement containing Boswellia serrata and Curcuma longa: a prospective randomized placebo-controlled study. Musculoskeletal surgery. 2015;99 Suppl 1:43-52. 